\title{
A case of perianal bowenoid papulosis: dermoscopic features and a review of previous cases
}

Yıldız Gürsel Ürün ${ }^{1 凶}$, Mustafa Ürün ${ }^{1}$, Sezin Fıçıcıoğlu ${ }^{1}$

${ }^{1}$ Department of Dermatology and Venereology, Faculty of Medicine, Trakya University, Edirne, Turkey.

\begin{abstract}
Bowenoid papulosis is an uncommon precancerous condition of the genitalia caused by oncogenic human papillomavirus types. It is seen in young, sexually active adults and histologically resembles Bowen's disease. Dermoscopy is useful in the diagnosis of both pigmented and non-pigmented skin lesions, but dermoscopic diagnostic accuracy criteria have not yet developed in diseases such as bowenoid papulosis and Bowen's disease. This case report analyzes the dermoscopic findings of bowenoid papulosis in the literature with the aim of increasing the frequency of use of dermoscopy in the diagnosis of bowenoid papulosis in clinical practice.
\end{abstract}

Keywords: bowenoid papulosis, precancerous conditions, dermoscopy, papillomavirus infections

Received: 13 July 2020 | Returned for modification: 25 September 2020 | Accepted: 11 November 2020

\section{Introduction}

Bowenoid papulosis (BP) corresponds with a multifocal intraepithelial neoplasia in the anogenital region and is most frequently seen in young adults. The male-to-female ratio for this condition is equal. Clinically, it most commonly presents with multiple welldemarcated hyperpigmented verrucous papules and plaques (1, 2). BP is frequently associated with human papillomavirus (HPV) infections, and in particular with HPV types 16 and 18, which are the oncogenic high-risk types (2).

Histopathologically, it is characterized by koilocytosis, enlarged polymorphic and hyperchromatic nuclei, and abnormal mitosis and hyper- or parakeratosis with a collection of melanin of various amount (1). Although the histopathologic findings are similar to Bowen's disease (BD), the distinction is based on differentiation, the degree of cellular atypia, and follicular involvement. In contrast to $\mathrm{BD}$, these histopathologic changes are more focal and less pronounced, and follicular involvement is not seen (3).

Dermoscopy is useful in the diagnosis of both pigmented and non-pigmented, or melanocytic and non-melanocytic, skin lesions by revealing the pigmentation pattern and vascular morphology, which cannot be seen by the naked eye. Recent studies have mainly focused on the dermoscopic separation of melanoma and melanocytic nevi. However, non-melanocytic pigmented lesions are common in clinical practice.

Herein, we describe the dermoscopic findings in a patient with perianal BP through a literature review of previously reported cases.

\section{Case report}

A 46-year-old woman presented with multiple, small, brown-gray papules on the perianal region with a duration of nearly 2 months (Fig. 1). Her HIV-1/2 tests and syphilis serology were negative, but she had been a hepatitis B carrier for 10 years. Two skin biopsies of the perianal region were performed to establish a differential diagnosis. A dermoscopic examination was also performed, which revealed brown-gray structureless areas with linearly distributed brown-gray dots and glomerular vessels in these homogeneous structures (Fig. 2). A microscopic examination revealed epidermis with varying degrees of hyperplasia and cytologic atypia with hyperchromasia and nuclear vesiculation, as well as abnormal mitosis (Fig. 3). The histological findings were multifocal, and no follicular involvement was observed. A real-time PCR testthe Cobas 4800 HPV test (Roche Diagnostics, GmbH, Mannheim, Germany)-was used to detect the HPV type. This resulted in the identification of HPV 18. Based on these clinical and histopathologic findings, the patient was diagnosed with BP, and topical imiquimod treatment was initiated. At the 3 rd week of treatment, treatment was stopped due to intense erythema in the perianal area. After the skin reaction became moderate, treatment with imiquimod cream was continued. At the end of the 2nd month, almost complete improvement was achieved in the lesions.

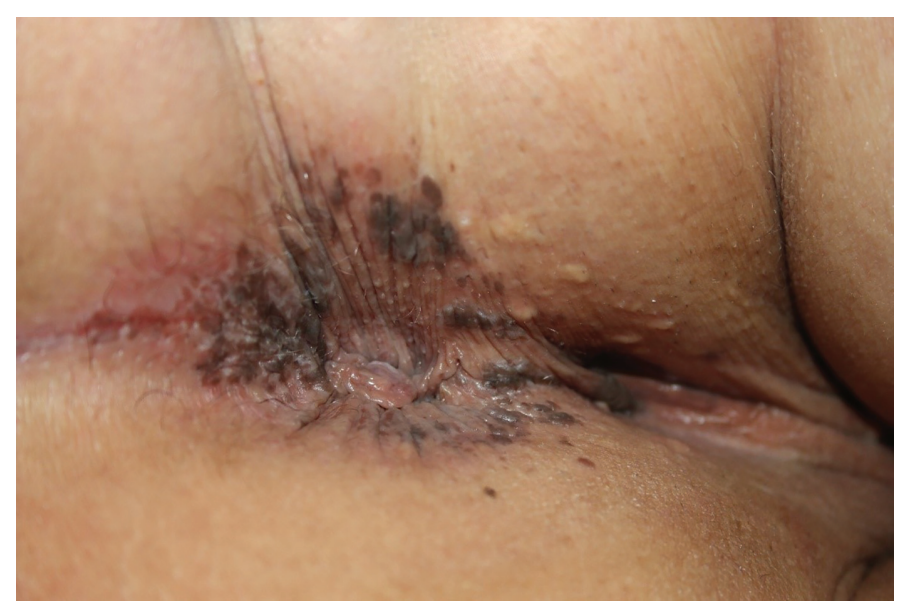

Figure 1 | Brown-gray papular lesions on the perianal region. 


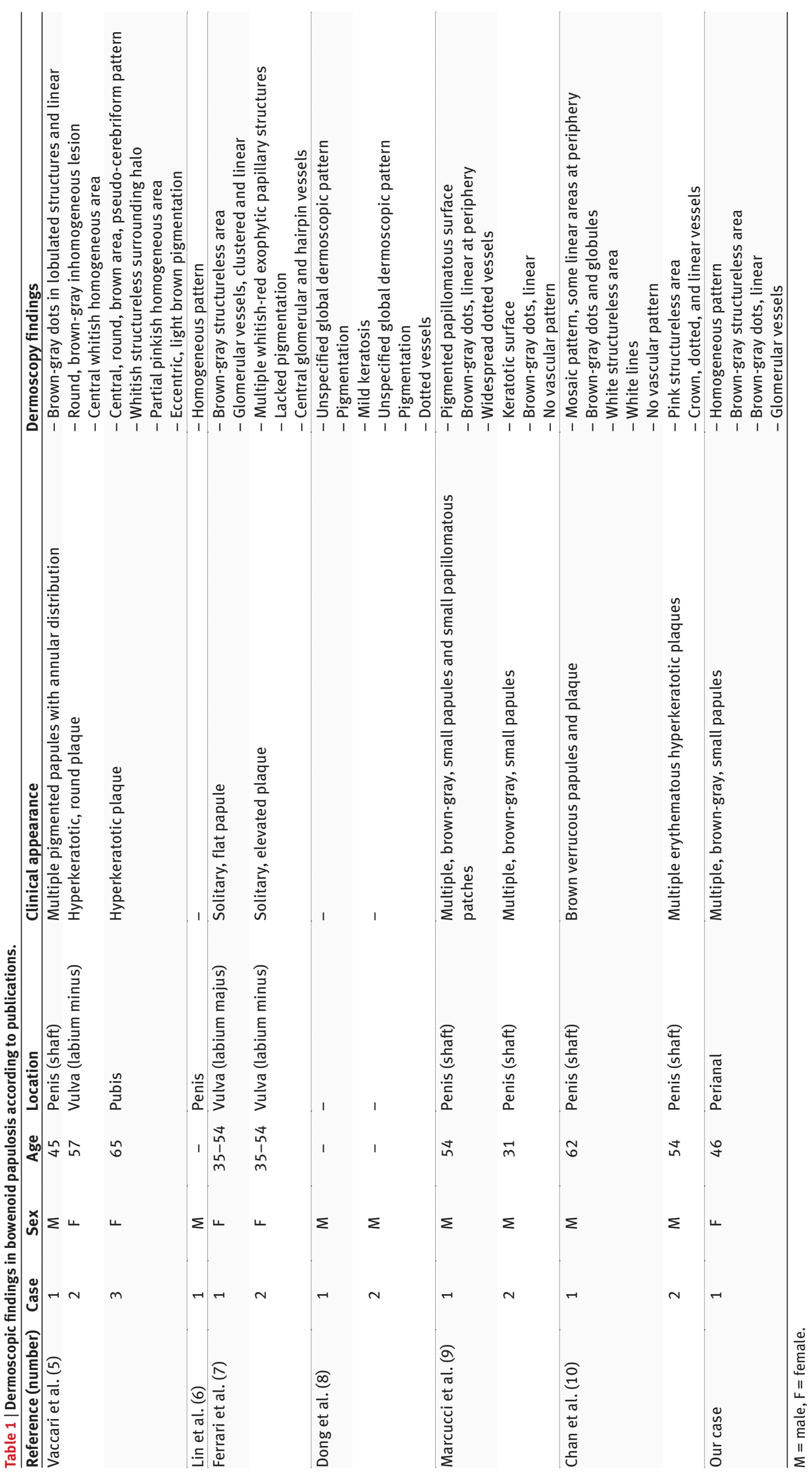




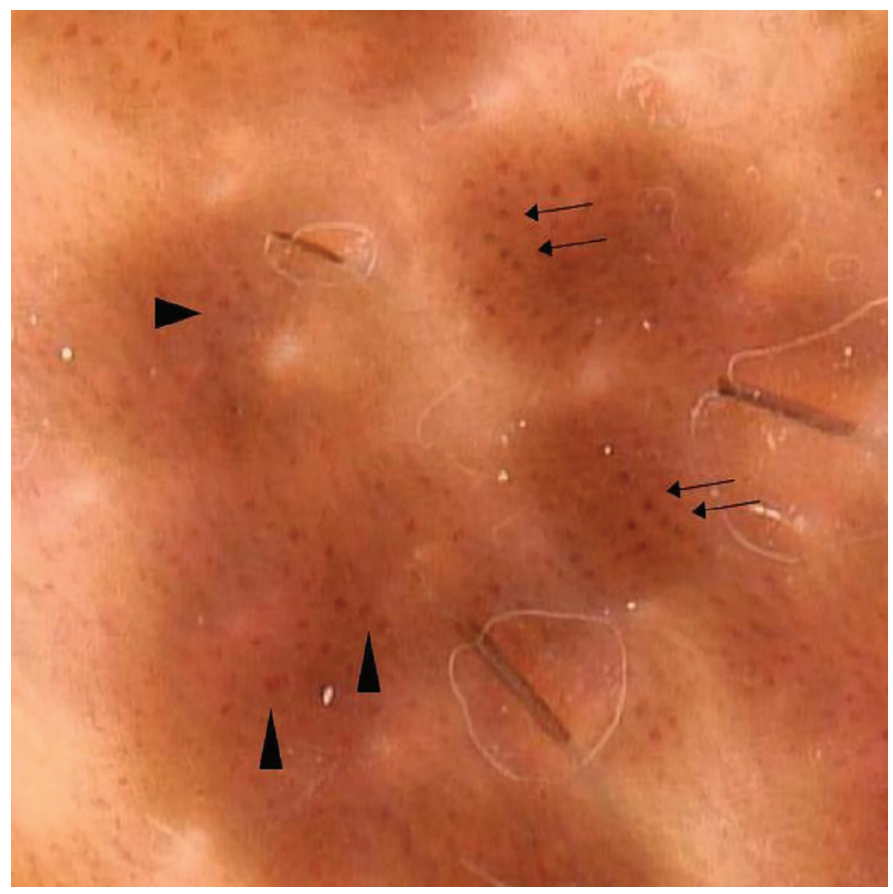

Figure 2 | Brown-gray dots arranged in a linear distribution (arrows) and widespread glomerular vessels (triangles) (original magnification 20x).

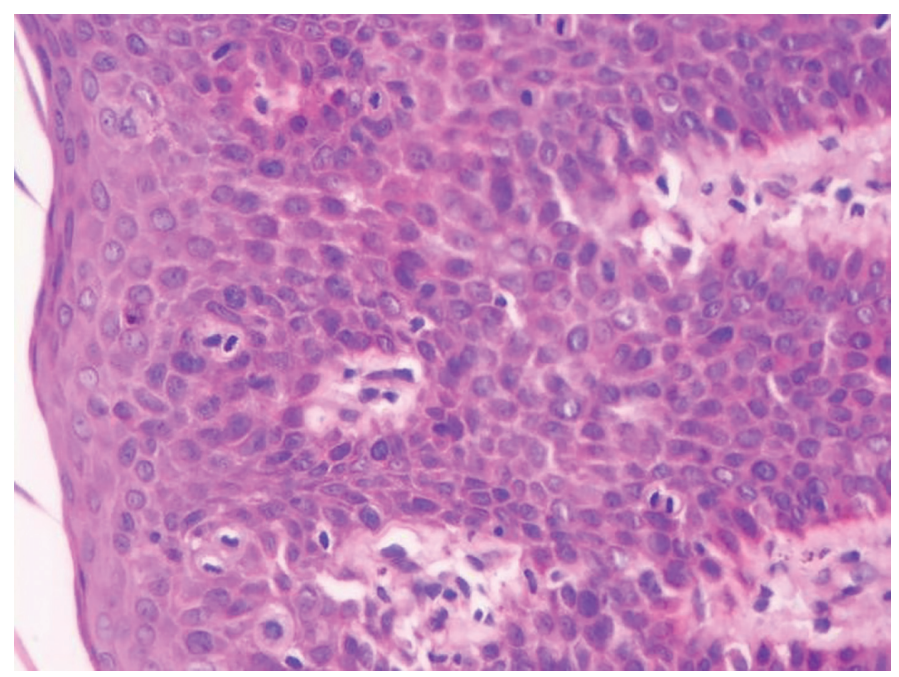

Figure 3 | Atypical and dyskeratotic keratinocytes with mitotic figures (H\&E, $40 x)$

\section{Discussion}

Although BP typically shows a benign prognosis, similar to genital warts, this condition histopathologically shares identical histolog- ical features with squamous cell carcinoma (SCC) in situ (1). The course of $\mathrm{BP}$ is variable in that the papules may regress, persist, recur, or progress to BD or invasive SCC in certain cases $(2,4)$. The risk of developing $\mathrm{BD}$ or SCC is higher in immunocompromised patients with BP (5). Because the likelihood of malignant transformation is between 1 and 2.6\%, early diagnosis and follow-up are critical (2). It is also worth noting that dermoscopy has recently gained importance during the early and differential diagnosis of BP.

Melanoma, pigmented melanocytic nevi, and pigmented nonmelanocytic lesions (genital warts, lichen planus, seborrheic keratosis, BD, basal cell carcinoma, and SCC) should be considered during the differential diagnosis of BP $(4,5)$. Dermoscopic diagnostic accuracy criteria have not yet developed in diseases such as BD, pigmented lichen planus, and BP. Nonetheless, dermoscopy can prove useful in differentiating BP from other conditions, such as genital warts, lichen planus, seborrheic keratosis, basal cell carcinoma, and even melanoma. Although it is rather difficult to distinguish BP from pigmented BD using dermoscopy, the patient's age and medical history can help differentiate between these two entities. Older age and chronic irritation anamnesis favor the diagnosis of pigmented BD (2). The final diagnosis of BP is histopathological (5).

Lesions are highly contagious, and patients with BP and their sexual partners should be monitored periodically because of the increased risk of developing SCC and cervical or vulvar neoplasia. Treatment of BP is recommended despite the low risk of malignant transformation (2). Therapeutic options include local destructive or ablative measures such as cryosurgery, electrodesiccation, laser vaporization, and surgical excision. Other modalities include topical use of ointments and creams containing 5-FU, podophyllin, retinoic acid, cidofovir, and imiquimod (1).

The dermoscopic findings of BP in studies published as case reports and small case series are summarized in Table 1 (5-10). Our findings are partially similar to previously reported cases. The most common dermoscopic findings include brown and gray dot structures with linear extension and glomerular and dotted vascular structures.

In summary, we analyzed the dermoscopic findings of BP in the literature with the aim of increasing the use of dermoscopy in the diagnosis of BP in clinical practice. However, because clinical BP lesions can be exophytic, warty, or papillary structures that are pigmented or skin-colored, and their dermoscopic appearance may differ from their clinical appearance, there are no specifically characterized dermoscopic findings of BP in the literature. Therefore, identification of different dermoscopic features in BP is required, as well as larger case series to help characterize these features.

\section{References}

1. Kutlubay Z, Engin B, Zara T, Tuzun Y. Anogenital malignancies and premalignancies: facts and controversies. Clin Dermatol. 2013;31:362-73.

2. Duncan KO, Geisse JK, Leffell DJ. Epithelial precancerous lesions. In: Goldsmith LA, Katz SI, Gilchrest BA, Paller AS, Leffell DJ, Wolff K, editors. Fitzpatrick's dermatology in general medicine. 8th ed. New York: McGraw-Hill; 2012. p. 1261-83.

3. Yu DS, Kim G, Song HJ, Oh CH. Morphometric assessment of nuclei in Bowen's disease and bowenoid papulosis. Skin Res Technol. 2004;10:67-70.

4. Shastry V, Betkerur J, Kushalappa. Bowenoid papulosis of the genitalia successfully treated with topical tazarotene: a report of two cases. Indian J Dermatol. 2009;54:283-6.

5. Vaccari S, Barisani A, Dika E, Fanti PA, D’Antuono A, Gaspari V, et al. Genital bowenoid papulosis: the variegated dermoscopic features. $G$ Ital Dermatol Venereol. 2018;153:595-7.
6. Lin J, Koga H, Takata M, Saida T. Dermoscopy of pigmented lesions on mucocutaneous junction and mucous membrane. Br J Dermatol. 2009;161:1255-6.

7. Ferrari A, Zalaudek I, Argenziano G, Buccini P, De Simone P, Silipo V, et al. Dermoscopy of pigmented lesions of the vulva: a retrospective morphological study. Dermatol. 2011;222:157-66.

8. Dong H, Shu D, Campbell TM, Frühauf J, Soyer HP, Hofmann-Wellenhof R. Dermatoscopy of genital warts. J Am Acad Dermatol. 2011;64:859-64.

9. Marcucci C, Sabban EC, Friedman P, Peralta R, Calb I, Cabo H. Dermoscopic findings in bowenoid papulosis: report of two cases. Dermatol Pract Concept. 2014;4:61-3.

10. Chan SL, Watchorn RE, Panagou E, Panou E, Ong EL, Heelan K, et al. Dermatoscopic findings of penile intraepithelial neoplasia: bowenoid papulosis, Bowen disease and erythroplasia of Queyrat. Australas J Dermatol. 2019;60:e201-7. 